\title{
Wear Behavior and Tribological Evolution of High Entropy Alloys
}

\author{
Sushil Kumar, Satpal Sharma
}

\begin{abstract}
High entropy alloy are equiatomic and nonequiatomic complex concentrated/ multicomponent alloys which are recognized due to their distinctive mechanical and triboligical properties. A unique combination of excellent mechanical and tribological properties of high entropy alloys makes them promising candidate for variety of industrial and structural applications. The wear resistance needs to be examined for these complex concentrated alloys as only few numbers of reports and investigation are available in the field of new advanced HEAs materials. In the current research work, we identified the crucial achievements and breakthrough in the wear and tribological investigations of high entropy alloy and HEA based composites in recent years. This review article investigates the tribological behaviors of multicomponent alloys /high entropy alloys and HEA based composites which play an important role and draw a considerable attention in the present era. In view of recent developments on tribological and wear related mechanisms of HEAs for different type of industrial and structural applications, microstructure, chemical compositions, and mechanical properties are explained and reviewed in this review article.
\end{abstract}

Index Terms- High entropy alloys, Wear behavior, Tribological properties, Mechanical Properties.

\section{INTRODUCTION}

The emergence and evolution of high entropy alloys and HEA based composites reported novel properties with improved mechanical performance compared to commercial alloys and traditional composites [1]. High entropy alloys based on the concept of incorporating five or more metallic elements ranging from 5 to 35 percent in atomic percentage to exhibit high configurational entropy of stable solid solutions for innovative applications. Recent advancements in the field of high entropy alloys with enhanced properties have captured the attention of researchers in developing hi tech future materials. The high entropy alloys (HEAs) have gained much popularity as a result of their vast range of mechanical, tribological and metallurgical properties over the conventional/ commercial alloys. The comprehensive effects of multicomponent alloys creates new possibilities to utilize them in variety of applications in different industrial and defence sectors, where light and strong material is always preferred [2]. The main benefits of complex concentrated alloys that they can generate vast variety of microstructures and can contributed crucially in the discovery of new scientific materials with attractive tribo-mechanical

Revised Version Manuscript Received on 10, September 2019.

Sushil Kumar,Research Scholar, Department of Mechanical India.(Email: sushil_eng@yahoo.com)

Satpal Sharma, Faculty, Department of Mechanical Engineering, Gautam Buddha University, Greater Noida, Uttar Pradesh, India.(Email: satpal@gbu.ac.in) Engineering, Gautam Buddha University, Greater Noida, Uttar Pradesh,

properties. [3]. In this manuscript, several combination of newly developed complex concentrated alloys concerning the tribological performance and wear related mechanisms discussed in the literature. The studies revealed the presence of hard particles in the complex microstructure is responsible for improve wear resistance in the family of high entropy alloys.

\section{FRICTION AND WEAR OF BEHAVIOR OF HEA UNDER DRY SLIDING CONDITION}

The high entropy materials fabricated and invented for specific applications must exhibit higher wear resistance at intense loading applications without showing deformation or fracture during implementation in longer periods. Poulia et al [4] examined the dry-sliding wear behavior of Mo20Ta20W20Nb20V20 of multicomponent alloy under different experimental conditions. The test analysis revealed high wear resistance of Mo20Ta20W20Nb20V20 when compared with a commercial super alloy. All the test results exhibited improved behavior of the high entropy alloy.Xiaotao et al [5] investigated the wear mechanism, microstructure and phase configuration of $\mathrm{A} 10.5 \mathrm{CoCrCuFeNiBx}$ high entropy alloy fabricated by vaccum arc melting in the argon atmosphere. The combination of hard borides and FCC matrix phase greatly enhanced the wear properties of the high entropy alloy. The analysis revealed that mechanism of wear is converted into oxidative wear from delamination wear while addition of boron content in the high entropy alloy. Yang et al [6] analyzed the effects of boron on microstructural and wear mechanism of FeCoCrNiAlBx high entropy alloy coatings synthesized by laser cladding technique. The coatings shows exceptional improved wear resistance by incorporating boron in the high entropy alloy coating. The addition of boron changed adhesive wear to abrasive wear and hardness also improved of high entropy alloy coating. Yadav et al [7] utilized the soft dispersoid such as $\mathrm{Pb}$ and $\mathrm{Bi}$ in the $\mathrm{AlCrFeMnV})$ and $(\mathrm{CuCrFeTiZn})$ high entropy alloy matrix. The CuCrFeTiZn)100-xPbx and (AlCrFeMnV)100-xBix high entropy composites fabricated through powder metallurgy process to analyzed wear behaviour at different test parameters. The results showed that introduction of soft dispersoid and high hardness significantly improved the microstructure of the HEA composites. Zhou et al [8] adopted the powder metallurgy route to investigate wear behavior and microstructural orientation of newly developed 
(FeCoCrNi)1-x(WC)x multicomponent composites followed by SPS technique. The presence of $\mathrm{Wc}$ particles and $\mathrm{Cr}$ carbide produced strengthening effect and combination of adhesive and abrasive wear mechanism obtained. Gasan and Lokcu [9] studied the microstructure, hardness and wear resistance of $\mathrm{AlxCoCrFeMo0.5Ni} \mathrm{(x=0-2)} \mathrm{HEAs} \mathrm{using}$ ball-on-disk testing machine. The results demonstrate that enhancement in percentage of aluminium improves the wear and tribological properties of the high entropy alloy. Yadav et al [10]studied microstructural and tribological properties of CuCrFeTiZn multicomponent alloy and (CuCrFeTiZn) 100-xPbx composites fabricated through MA and SPS technique. The high entropy alloy with $10 \% \mathrm{~Pb}$ showed 95 percent less wear than base HEA alloy. The high entropy alloy containing $\mathrm{Pb}$ proved excellent candidate for bearing applications.Liu et al [11] fabricated HEA composites using Fe50Mn30Co10Cr10 as matrix and nano particulates of graphene as reinforcement material. A friction and wear tester was utilized to execute the wear tests at a room temperature and analysis showed that the coefficients of friction (COF) of high entropy composites were reduced drastically in comparison Fe50Mn30Co10Cr10 matrix HEA. The results revealed enhancement in the tribological properties of the fabricated composites when compared to the matrix. Löbel et al [12] fabricated equimolar AlCoCrFeNiTi HEA and AlCoCrFeNiTi composites reinforced with solid lubricant MoS2 in order to assess wear resistance and reduced coefficient of friction. AlCoCrFeNiTi HEA reinforced with $10 \mathrm{wt} \% \mathrm{MoS} 2$ exhibit improved wear resistance and reduced COF at different temperatures. The high entropy composites are considered decisive material for bearing and other wear protection applications Yadav et al [13] analyzed the tribological behavior of AlCrFeMnV BCC alloy as a matrix along with $\mathrm{Bi}$ reinforced with TiB2 solid lubricants. Addition TiB2 and uniformly dispersed Bi particles leads to $85 \%$ decrease in wear rate compared to base alloy. Softening and melting Bi particles acts as a lubricating film between the sliding bodies Mathiou et al [14] examined the microstructural and wear behavior of MoTaNbZrTi high-entropy alloy employing a ball-on disc machine at room temperature at three different sliding distances. The wear properties of complex concentrated alloy were compared with different high entropy alloys and results showed the exceptional improvements in tribological properties of MoTaNbZrTi high entropy alloy.Nong et al [15] fabricated $\mathrm{AlCrFeNiTi}$ and AlCrFeNiTiMn0.5 through vaccum arc melting in argon atmosphere and reported enhancement in wear and oxidation resistance of high entropy alloys. It is obvious from the studies, that with dissimilarity in grain structure, phase constitution and elemental compositions of solid solutions of HEA, different tribological and metallurgical properties can be achieved.

\section{EFFECT OF ENVIRONMENTAL CONDITIONS ON WEAR MECHANISM \& RESULTS}

Although, high entropy alloys showed the potential of higher strength to weight ratio, while exhibting excellent wear properties, but a watchful recognition of the conditions through investigation and understanding of tribological behavior is a main challenge for selection of elements and their evolution. Duan et al [16] studied the tribological mechanism of $\mathrm{AlCoCrFeNiCu} \mathrm{HEA} \mathrm{using} \mathrm{pin-on-disc} \mathrm{tests} \mathrm{by}$ submerging the specimen in the lubricant oil and hydrogen peroxide solution. The results confirmed that wear mechanism of $\mathrm{AlCoCrFeNiCu}$ affected by environmental conditions and showed low coefficient of friction against $\mathrm{Si}_{3} \mathrm{~N}_{4}$ disk under lubricant oil. Yu et al [17] investigated tribological properties and wear behavior of the $\mathrm{AlCoCrCuFeNi}$ and $\mathrm{AlCoCrFeNiTi0.5} \mathrm{HEAs} \mathrm{against} \mathrm{ZrO} 2$, $\mathrm{SiC}$ and $1 \mathrm{Cr} 18 \mathrm{Ni9Ti}$ steel discs under hydrogen peroxide solution. High wear loss and COF observed in AlCoCrFeNiTi0.5/1Cr18Ni9Ti steel discs whereas better wear properties achieved in $\mathrm{AlCoCrFeNiTi0.5}$ alloy/SiC discs combination. Yipin et al [18] utilized hot sintering process to fabricate $\mathrm{AlCrFeNi}$ high entropy alloy coating on the surface of Q235 steel. Coating formation mechanism, dry sliding and oil lubrication wear tests, wear morphology of HEA films were studied. AlCrFeNi high entropy alloys coating showed excellent wear resistance while performing dry sliding and oil lubrication tests. Ma et al studied [19] the environmental effect on wear behavior of $\mathrm{Al} 0.6 \mathrm{CoCrFeNi}$ HEA under different conditions. The results demonstrated higher wear resistance in sea water compared to that in acid rain, ambient air and deionized water. The wear mass loss is higher in ambient air conditions compared to that in liquid conditions. The wear mechanisms in ambient dry conditions and deionized water conditions were abrasive wear and oxidative wear, delamination and adhesive wear. Yu et al [20] studied the tribological mechanisms of AlCoCrFeNi-M high entropy alloys at different sliding speeds under under $90 \%$ $\mathrm{H} 2 \mathrm{O} 2$ solution. The studies reported the supremacy in the triblogical behaviour and wear related mechanism of high entropy alloys. Yua et al [21] examined the wear mechanism of $\mathrm{CoCrFeNiNbx}$ high entropy alloy to utilize in high temperature applications. The effect of friction, wear and high temparture on the structure of $\mathrm{CoCrFeNiNbx}$ high entropy alloy analyzed. At $400 \mathrm{C}$, abrasive wear is changed into adhesive wear then at $600 \mathrm{c}$ it is converted into oxidation wear and mechanical wear. Jin et al [22] synthesized the $\mathrm{FeNiCoAlCu} \mathrm{HEA}$ coating through laser technique to analyze the wear behaviour at high temperature. Laser cladded $\mathrm{FeNiCoAlCu}$ consist of dendrite microstructure with FCC and BCC phases governed by abrasive wear and oxidative wear. The emergence of oxide film and enhanced thermal stability revealed the improved wear resistance of HEA coating at high temperature. Joseph et al [23] investigated the tribological mechanism of $\mathrm{CoCrFeMnNi}$ and AlxCoCrFeNi high entropy alloys at different temperature range and results were compared with AISI 304 and Inconel 718. AlCoCrFeNi HEA showed excellent wear resistance compared to other HEAs test specimen at different temperature range

\section{WEAR RESISTANCE OF HEA CLADDING/ COATING / SUBSTRATE}

Tüten et al [24] investigated the sliding wear and coefficient of friction of equimolar TiTaHfNbZr high entropy thin films deposited on the Ti6Al4V substrates for orthopedic 
applications. The results demonstrated improved surface protection of HEA films against wear and cracking and can be efficiently utilized in knee and hip joints. Chen et al [25] fabricated Al0.6TiCrFeCoNi HEA coating through high-velocity-oxygen-fuel (HVOF) spraying in order to compute the fracture hardness and phase configurational detail. The wear behavior of coating was analyzed by using pin on disc tribometer under different temperature conditions. Huang et al [26] reported that wear properties of AZ91D magnesium alloys were improved by fabrication of $\mathrm{Cu} 0.9 \mathrm{NiAlCoCrFe}$ high entropy alloy (HEA) coating through laser cladding method. The results demonstrated that BCC solid solution phase of HEA films are mainly responsible in improvement of wear properties of HEA. The obtained mechanisms from the results were found different in HEA coating and the AZ91D substrate. The HEA coating influenced by oxidative abrasive wear and the AZ91D substrate undergoes with adhesive and abrasive wear. Wang et al [27] studied wear properties and microstructure of CuNiSiTiZr high entropy alloy synthesized by vaccum arc melting deposited on TC11 titanium alloy through electrospark CNC deposition technique. The BCC solid solution of the coating exhibit enhanced wear behavior and hardness compared TC11 substrate. Huo et al [28] analyzed the microstructural and wear properties of $\mathrm{CoCrFeMnNbNi}$ high entropy alloy coating was fabricated on AISI304 steel through TIG cladding technique. The microhardness and wear properties of the CoCrFeMnNbNi high entropy significantly enhanced under dry sliding wear due to the presence hard laves phase and FCC solid-solution phase. Yipin et al [29] implemented hot sintering process to fabricate $\mathrm{AlCrFeNi}$ high entropy alloy coating on the surface of Q235 steel. Coating formation mechanism, dry sliding and oil lubrication wear tests, wear morphology of HEA films were studied. $\mathrm{AlCrFeNi}$ multicomponent solid solution coating demonstrated excellent improvement in wear behavior in both dry sliding and oil lubrication tests due to presence of to its hard phases. Cai et al [30] studied wear resistance, microstructure and phase composition of the Ni-Cr-Co-Ti-V high entropy alloy coating through combination of laser cladding and laser remelting processes. The results demonstrate that remelted HEA coating exhibit better wear performance due to high hardness compared to laser cladded coatings. Low wear losses, Ti rich phases and high COF of BCC solid solution phase improved the wear resistance of the combination of laser cladding and laser remelting of HEA coating. Ye et al[31] implemented nanoscratch method to evaluate the friction and wear behaviour of TiZrHfNb high entropy alloy at ramping and constant load. TiZrHfNb high entropy alloy showed excellent hardness/strength and wear resistance on ramping and constant load modes. The test outcomes revealed that developed multicomponent alloy consist of reduced coefficient of friction and improved wear properties can be employed in various industrial applications. Minghong et al [32] investigated wear properties and microstructure of cast and annealed AlCoCrFeNiTi0.5 high entropy alloy synthesized by laser cladding technique. The investigations showed wear mass loss of HEA coatings minimized upto $92.5 \%$ and wear width decreased upto $50 \%$ compared to cast specimens. The BCC structure with solid solution formed on the annealed surface remarkably improved the wear properties and microstructure of HEA coatings due to solute and precipitation strengthening.

\section{CONCLUSION}

The reported improvements in the mechanical, microstructural and tribological properties of complex concentrated alloys prove them significant attractive materials in variety of structural and industrial applications. Several compositions of HEAs exhibit enhanced wear resistance compared to traditional alloys or composites in different operating conditions. Understanding of the surface characteristics of multicomponent alloys is very important to know the wear related properties and to utilise them in commercial applications. The wear properties of complex concentrated alloys/ HEAs alloys are further required to examine for the evolution in advanced next generation materials.

\section{REFERENCES}

1. J. W. Yeh, S. K. Chen, S. J. Lin, J. Y. Gan, T. S. Chin, T. T. Shun, C. H. Tsau, S. Y. Chang, Advanced Engineering Materials, 6 (2004), pp 299-303.

2. T. K. Chen, T. T. Shun, J. W. Yeh, M. S. Wong, Surface \& Coatings Technology, 188-189 (2004), pp 193-200.

3. Zhang Luchan, Xiang Yang, Han Jian, Srolovitz David, Acta Materialia, (2018), pp. 1-16.

4. A. Poulia, E. Georgatis, A. Lekatou, A. E. Karantzalis, International Journal of Refractory Metals and Hard Materials, Vol 57 (2016) pp. 50-63.

5. Liu Xiaotao, Lei Wenbin, Ma Lijuan, Liu Jinling, Liu Jing, Cui Jianzhong, Rare Metal Materials and Engineering, Vol. 45(9)(2016), pp 2201-2207.

6. DanYang Lin,Nannan Zhang,Bin He, GuangWei Zhang,Yue Zhang, De Yuan Li, Journal of Iron and Steel Research, International, Vol. 24(2017), pp 184-189.

7. Surekha Yadav, Arvind Kumar, Krishanu Biswas, Materials Chemistry and Physics, (2017), pp 1-11.

8. Rui Zhou, Gang Chen, Bin Liu, Jiawen Wang, Liu liu Han, Yong Li, International Journal of Refractory Metals and Hard Materials; Vol. 75 (2018), pp. 56-62.

9. Hakan Gasan and Ersu Lokcu, Journal of Science and Technology; Vol. 7(4) (2017) pp. 63-67.

10. SurekhaYadav, S. Sarkar, AkashAggarwal, Arvind Kumar and Krishanu Biswas, Wear, (2018), pp 1-11.

11. Xinyu Liu, Shuqiang Zhou, Yi Xu, Materials Letters; Vol. 233 (2018) pp 142-145.

12. Martin Lobel, Thomas Lindner and Thomas Lampke, Materials; Vol. 11(11) (2018), pp. 1-11.

13. Surekha Yadav, Akash Aggrawal, Arvind Kumar, Krishanu Biswas, Tribology International; Vol. 132 (2019) pp. 62-74.

14. C. Mathiou, A. Poulia, E. Georgatis, A. Karantzalis, Materials Chemistry and Physics; Vol. 210(2018); pp. 126-135

15. Zhi-Sheng Nong, Yu-Nong Lei, Jing-Chuan Zhu, Intermetallics; Vol. 101 (2018), pp. 144-151.

16. Haitao Duan, YongWu, Meng Hua,Chengqing Yuan, Ding Wang, Jiesong Tu, Hongchao Kou, Jian Li, Wear; 297 (2013), pp. 1045-1051.

17. Yuan Yu, Jun Wang, Jun Yang, Zhuhui Qiao, Haitao Duan, Jinshan Li, Jian Li, Weimin Liu, Tribology International (2018), pp 1-5 
18. Tang Yipin, Wang Shouren, Sun Bin, Wang Yan and Qiao Yang, Surface Review and Letters, Vol. 23(4) (2016), pp. $1-12$.

19. Xiaoguang Ma, Jian Chen, Xianhui Wang, Yanjin Xu, Yujie Xu, Journal of Alloys and Compounds 795 (2019), pp. 45-53.

20. Yuan Yu, Jun Wang, Jun Yang, Zhuhui Qiao, Haitao Duan, Jinshan Li, Jian Li, Weimin Liu, Tribology International (2018), pp. 22-26

21. Y. Yu, F. He, Z. Qiao, Z. Wang, W. Liu, J. Yang, Journal of Alloys and Compounds (2018), pp. 19-25.

22. G. Jin, Z. Cai, Y. Guan, X. Cui, Z. Liu, Y. Li, M. Dong, D. Zhang, Applied Surface Science (2018), pp. 72-79.

23. Jithin Joseph, Nima Haghdadi, Karl Shamlaye, Peter Hodgson, Matthew Barnett and Daniel Fabijanic, Wear (2019), pp. 1-15.

24. N. Tuten, D. Canadinc, A. Motallebzadeh, B. Bal, Microstructure and Tribological Properties of Intermetallics, Vol. 105 (2019), pp. 99-106.

25. Lijia Chen, Kirsten Bobzin, Zheng Zhou, Lidong Zhao, Mehmet Ote, Tim Konigstein, Zhen Tan, Dingyong He, Surface and Coatings Technology, Vol. 358 (2019), pp. 215-222.

26. Kaijin Huang, Yuyue Wang and Xin Lin; Advanced Materials Research; Vol. 989-99(2014), pp. 246-250.

27. Xiao-Rong Wang, Zhao-Qin Wang, Peng He, Tie-Song Lin, Surface \& Coatings Technology; (2015), pp. 9-14.

28. Wen-yiHuo, Hai-fang Shi, Xin Ren, Jing-yuan Zhang, Advances in Materials Science and Engineering; Volume (2015), pp.1- 5 .

29. Tang Yipin, Wang Shouren, Sun Bin, Wang Yan and Qiao Yang, Surface Review and Letters; Vol. 23(4) (2016), pp. 1-12.

30. Zhaobing cai, Xiufang Cui,Zhe Liu, Yang Li, Meiling Dong, Guo Jin, Optics and Laser Technology (2017), pp. 18-23.

31. Y.X. Ye, C.Z. Liu, H. Wang, T.G. Nieh, Acta Materialia, Vol. 147 (2018), pp. 78-89.

32. Sha Minghong, Zhang Li, Zhang Junwei, Li Na, Li Taizeng, Wang Ning, Rare Metal Materials and Engineering, Vol 46(5) (2017), pp. 1237-1240. 